\title{
A composite estimator for stratified two stage cluster sampling
}

\author{
Sang Eun Lee ${ }^{a}$, Pu Reum Lee ${ }^{b}$, Key-II Shin ${ }^{1, b}$ \\ ${ }^{a}$ Department of Applied and Information Statistics, Kyonggi University, Korea \\ ${ }^{b}$ Department of Statistics, Hankuk University of Foreign Studies, Korea
}

\begin{abstract}
Stratified cluster sampling has been widely used for effective parameter estimations due to reductions in time and cost. The probability proportional to size (PPS) sampling method is used when the number of cluster element are significantly different. However, simple random sampling (SRS) is commonly used for simplicity if the number of cluster elements are almost the same. Also it is known that the ratio estimator produces a good performance when the total number of population elements is known. However, the two stage cluster estimator should be used if the total number of elements in population is neither known nor accurate. In this study we suggest a composite estimator by combining the ratio estimator and the two stage cluster estimator to obtain a better estimate under a certain population circumstance. Simulation studies are conducted to compare the superiority of the suggested estimator with two other estimators.
\end{abstract}

Keywords: post weight adjustment, jackknife method, linear combination, ratio estimator, enumerated district

\section{Introduction}

Stratified two stage cluster sampling is widely used to reduce time and cost for the effective estimation of total and variance. However, when reducing the cost, the accuracy of estimates will not be good if a small number of clusters are selected and many units in each selected cluster are sampled. Furthermore, the accuracy of estimates deteriorates when cluster sizes are very different and simple random sampling method is used for cluster selection. For instance, in a household survey, if we set the cluster with district named Dong, Yup, Myun and select a small number of clusters using a SRS method, the accuracy of the estimates is not guaranteed due to the differences in cluster sizes. A common solution for this issue is to use a PPS method with known cluster sizes and the total number of population elements. However, a stratified two stage cluster sampling method with SRS is frequently adopted if the differences of sizes of each cluster are small. For instance, in household survey, census is mostly used as a sampling frame which is formed by clusters called as enumerated district (ED) with including about sixty households and the stratified two stage cluster sampling method with SRS is commonly used.

However, the stratified two stage cluster sampling with SRS is not an appropriate method if we select a rather small number of clusters with different sizes because there may exist a difference between the total sum of design weights and the total number of population elements. Therefore,

\footnotetext{
${ }^{1}$ Corresponding author: Department of Statistics, Hankuk University of Foreign Studies, 81 Oedae-ro, Mohyeon-myeon, Cheoin-gu, Yongin-si, Gyeonggi-do 17035, Korea. E-mail: keyshin@hufs.ac.kr

Published 31 January 2016 / journal homepage: http://csam.or.kr

(c) 2016 The Korean Statistical Society, and Korean International Statistical Society. All rights reserved.
} 
we need to eliminate the difference for more accurate estimates. For that adjustment, the size of the population needs to be known and the calibration adjustment can be applied. Here the obtained estimator using the calibration adjustment is known as the ratio estimator. For this reason, the ratio estimator which utilizes the exact number of population elements has better results than the other estimator obtained by a usual two stage cluster estimation.

However, getting the exact size of population is very difficult in practice. For instance, Census on Establishment in Korea is commonly used as a sampling frame. However, the information from that census was generally obtained two years prior. Hence, we suggest a composite estimator when the cluster sizes are not exact but with minor differences and a simple random sampling used to select clusters. A composite estimator is obtained by combining the two stage cluster estimator and the ratio estimator. To calculate the composite estimator, two weights on each estimator need to be calculated and obtained by one of the popular methods in Rao (2003). The variance estimate is known for the two stage cluster estimation method. The different clusters sizes then indicate an approximate variance estimator of the ratio estimator as illustrated in Cochran (1977).

This study used a Jackknife method (one of the popular replication variance estimation methods) for the ratio estimator since the variance estimate of the ratio estimator is approximately obtained. The weights which are the coefficients of the composite estimator are calculated using variances estimated in each estimation method.

In this paper, the two stage cluster sampling method will be explicitly mentioned and the composite estimator, which combines the ratio and the two stage cluster estimator as stated in Section 2. Also calculating the coefficients of the composite estimator (called as weights) is explained, especially the delete one cluster Jackknife variance estimation method for the ratio estimator is focused in Section 3. In Section 4, some simulation results are compared and a real data analysis is conducted using Taxi Company data in Section 5. The summary and conclusion are stated in Section 6.

\section{Two stage cluster sampling}

\subsection{Stratified two stage cluster sampling}

Stratified two stage cluster sampling method is a sampling technique to obtain an efficient estimation by selecting a part of elements in selected clusters. Here are the notations in this study. $L$ is the number of strata in population, $N_{h}$ is the number of clusters of population and $n_{h}$ is the number of sample clusters in stratum $h, M_{h i}$ is the number of elements in $i^{\text {th }}$ cluster, $h^{\text {th }}$ stratum in population and $m_{h i}$ is the size of sample units in $i^{\text {th }}$ sampled cluster, $h^{\text {th }}$ stratum. Therefore the total number of clusters in population is $N=\sum_{h=1}^{L} N_{h}$, the total number of elements of population is $M_{0}=\sum_{h=1}^{L} M_{h}$ and the total number of elements of population in stratum $h$ is $M_{h}=\sum_{i=1}^{N_{h}} M_{h i}$. Following estimators are well explained in Cochran (1977).

\subsubsection{Stratified two stage cluster estimation}

In stratified two stage cluster sampling with $L$ strata, the estimator of total, $\hat{Y}^{C}$ is defined by

$$
\hat{Y}^{C}=\sum_{h=1}^{L} \frac{N_{h}}{n_{h}} \sum_{i=1}^{n_{h}} \frac{M_{h i}}{m_{h i}} \sum_{j=1}^{m_{h i}} y_{h i j},
$$

where $y_{h i j}$ is the $j^{t h}$ observation in $i^{t h}$ cluster, stratum $h$. Now the unbiased variance estimate of the 
estimator, $\hat{Y}^{C}$ is as follows.

$$
\hat{V}\left(\hat{Y}^{C}\right)=\sum_{h=1}^{L}\left[\frac{N_{h}^{2}}{n_{h}}\left(1-f_{1 h}\right) \frac{\sum_{i=1}^{n_{h}}\left(\hat{Y}_{h i}-\overline{\hat{Y}}_{h}^{C}\right)^{2}}{n_{h}-1}+\frac{N_{h}}{n_{h}} \sum_{i=1}^{n_{h}} M_{h i}^{2}\left(1-f_{2 h i}\right) \frac{s_{2 h i}^{2}}{m_{h i}}\right],
$$

where $\hat{Y}_{h i}=M_{h i} \bar{y}_{h i}, \bar{y}_{h i}=m_{h i}^{-1} \sum_{j=1}^{m_{h i}} y_{h i j}, \overline{\hat{Y}}_{h}^{C}=n_{h}{ }^{-1} \sum_{i=1}^{n_{h}} M_{h i} \bar{y}_{h i}, f_{1 h}=n_{h} / N_{h}, f_{2 h i}=m_{h i} / M_{h i}$ and $s_{2 h i}{ }^{2}=\sum_{j=1}^{m_{h i}}\left(y_{h i j}-\bar{y}_{h i}\right)^{2} /\left(m_{h i}-1\right)$, the variance estimator for $i^{t h}$ cluster in $h^{\text {th }}$ stratum.

\subsubsection{Ratio estimator of stratified two-stage cluster sampling}

The ratio estimator is known as one of the calibration estimates in stratified two stage cluster sampling with $L$ strata. We assume that the number of elements in each population stratum, $M_{h}$ is known. Then the estimator of total, $\hat{Y}^{R}$ is:

$$
\hat{Y}^{R}=\sum_{h=1}^{L} \frac{M_{h}}{\sum_{i=1}^{n_{h}} M_{h i}} \sum_{i=1}^{n_{h}} \frac{M_{h i}}{m_{h i}} \sum_{j=1}^{m_{h i}} y_{h i j} .
$$

We use the additional information of the number of elements in stratum $h, M_{h}$ and may have more accurate estimate of total than that of (2.1). The usual variance estimator of $\hat{Y}^{R}$ is:

$$
\hat{V}\left(\hat{Y}^{R}\right)=\sum_{h=1}^{L}\left[\frac{N_{h}^{2}}{n_{h}} \frac{\sum_{i=1}^{n_{h}} M_{h i}^{2}\left(\overline{y_{h i}}-\hat{y_{h}}\right)^{2}}{n_{h-1}}\left(1-f_{1 h}\right)+\frac{N_{h}}{n_{h}} \sum_{i=1}^{n_{h}} M_{h i}^{2}\left(1-f_{2 h i}\right) \frac{s_{2 h i}{ }^{2}}{m_{h i}}\right] .
$$

Here $\hat{\overline{y_{h}}}=n_{h}{ }^{-1} \sum_{i=1}^{n_{h}} \overline{y_{h i}}$ and all other definitions are the same as in (2.2).

\subsection{Comparison of the estimators}

Now the sum of the design weight $\hat{w}$ can be obtained by plugging 1 into Equation (2.1) instead of $y_{h i j}$. That is $\hat{w}=\sum_{h=1}^{L} N_{h} / n_{h} \sum_{i=1}^{n_{h}} M_{h i}$.

If the size of population in stratum $h, M_{h}$ is known, then the ratio estimator can be used. If not, or inaccurate information, the value of $M_{h}$ must be estimated. The estimate of the size of population in stratum $h$ is as follows:

$$
\hat{M}_{h}=\frac{N_{h}}{n_{h}} \sum_{i=1}^{n_{h}} M_{h i}=N_{h} \hat{\bar{M}}_{h}
$$

Therefore if there are some problems of estimating the mean of size of each cluster, obviously the estimate of total should be worse. That is, if $N_{h} / n_{h}$ and $M_{h} / \sum_{i=1}^{n_{h}} M_{h i}=\sum_{i=1}^{N_{n}} M_{h i} / \sum_{i=1}^{n_{n}} M_{h i}$ are identical or at least similar, then the estimated value using (2.1) can be used.

Now assume that $M_{h i}$ is random and $M_{h i} \sim\left(\bar{M}_{h}, \sigma_{h}^{2}\right)$ with $\bar{M}_{h}$, mean of the number of elements of cluster and $\sigma_{h}^{2}$, the variance of $M_{h i}$. Then $\hat{\bar{M}}_{h}=n_{h}{ }^{-1} \sum_{i=1}^{n_{h}} M_{h i} \sim\left(\bar{M}_{h}, \sigma_{h}^{2} / n_{h}\right)$. Therefore if small number of sample clusters is used or $\sigma_{h}^{2}$ is large, then the accuracy of estimate of total obtained by the two stage cluster estimator becomes worse. 


\section{Suggested composite estimator}

\subsection{The composite estimator}

Practically the number of elements in each cluster is hardly the same. Of course, EDs in census have about 60 households and therefore $\sigma_{h}^{2} \approx 0$ and $\hat{M}_{h}$ can be estimated close to the true value.

However, for instance, EDs in Agriculture census have the different cluster sizes. In addition, the number of sample clusters is rather small in most of cases. Census cannot be used as sampling frame if the survey is not for official statistics. In those cases, the accuracy of the two stage cluster estimator can be declined.

To overcome this situation, we suggest a new composite estimator, $\hat{Y}_{C P}$, which combines two estimators $\hat{Y}_{C}$ and $\hat{Y}_{R}$.

The suggested linear composite estimator is defined by

$$
\hat{Y}^{C P}=\alpha \hat{Y}^{C}+(1-\alpha) \hat{Y}^{R},
$$

where $\alpha$ is the weight of the suggested composite estimator.

Now the estimated value of $\alpha$ can be obtained by

$$
\hat{\alpha}=\frac{\widehat{\operatorname{MSE}}\left(\hat{Y}^{R}\right)}{\widehat{\operatorname{MSE}}\left(\hat{Y}^{R}\right)+\widehat{\operatorname{MSE}}\left(\hat{Y}^{C}\right)} \approx \frac{\hat{V}\left(\hat{Y}^{R}\right)}{\hat{V}\left(\hat{Y}^{R}\right)+\hat{V}^{\left(\hat{Y}^{C}\right)}} .
$$

For more details of the estimated value of the weight $\alpha$, see Rao (2003) or Hwang and Shin (2013). Here $\hat{V}\left(\hat{Y}^{C}\right)$ and $\hat{V}\left(\hat{Y}^{R}\right)$ can be calculated using (2.2) and (2.4). However, we use the replication variance estimation, especially the Jackknife variance estimator since the variance estimator (2.4) is not unbiased.

\subsection{Jackknife variance estimator}

From Cochran (1977), we have that the variance estimate of the ratio estimator in stratified two stage cluster sampling is obtained approximately. So that in this study we use the delete one cluster Jackknife variance estimation method. This method is also used in Lee et al. (2015). The Jackknife variance method is a commonly used non-parametric methods that can reduce bias. Quenouille (1949) suggested at first and Tukey (1958) used this for variance estimation. More details are found in Wolter (1985).

The general set up for the Jackknife variance method is as follows. First using the size of $n$ samples, $Y_{1}, Y_{2}, \ldots, Y_{n}$, we can calculate $\hat{\theta}=f\left(Y_{1}, Y_{2}, \ldots, Y_{n}\right)$, an estimator of parameter $\theta$. Then similarly after deleting the $k^{\text {th }}$ element, the estimator deleted one element can be obtained as below.

$$
\hat{\theta}_{n(k)}=f\left(Y_{1}, Y_{2}, \ldots, Y_{k-1}, Y_{k+1}, \ldots, Y_{n}\right) .
$$

The Jackknife variance estimator is:

$$
V\left(\hat{\theta}_{J K}\right)=\frac{(n-1)}{n} \sum_{i=1}^{n}\left(\hat{\theta}_{n(k)}-\overline{\hat{\theta}}_{n(k)}\right)^{2},
$$

where $\overline{\hat{\theta}}_{n(k)}$ is the mean of $\hat{\theta}_{n(k)}$.

Now the delete one cluster Jackknife method which is used in this study is that deleting $k^{\text {th }}$ cluster is used instead of deleting one element. Using delete one cluster Jackknife variance method, the variance estimator of the ratio estimate is as follows. 
First from (2.3), the estimator of total of stratum $h$ is $\hat{Y}_{h}^{R}=\left(M_{h} / \sum_{i=1}^{n_{h}} M_{h i}\right) \sum_{i=1}^{n_{h}} M_{h i} / m_{h i} \sum_{j=1}^{m_{h i}} y_{h i j}$. After deleting $k^{\text {th }}$ cluster, the estimator of total of stratum $h$ is

$$
\hat{Y}_{h(k)}^{J K}=\frac{M_{h}}{\sum_{i=1}^{n_{h(k)}} M_{h i}} \sum_{i=1}^{n_{h(k)}} \frac{M_{h i}}{m_{h i}} \sum_{j=1}^{m_{h i}} y_{h i j},
$$

where

$$
n_{h(k)}= \begin{cases}n_{h(k)}=n_{h}-1, & \text { if } k \in \text { clusters in stratum } h, \\ n_{h(k)}=n_{h}, & \text { otherwise. }\end{cases}
$$

Now the estimator of total is $\hat{Y}_{(k)}^{J K}=\sum_{h=1}^{L} \hat{Y}_{h}(k)^{J K}$ and from (3.5) the Jackknife variance estimator is:

$$
V\left(\hat{Y}^{J K}\right)=\frac{(n-1)}{n} \sum_{k=1}^{n}\left(\hat{Y}_{(k)}^{J K}-\overline{\hat{Y}}_{(k)}^{J K}\right)^{2}
$$

where $n=\sum_{h=1}^{L} n_{h}$ is the total number of sample clusters and $\overline{\hat{Y}}_{(k)}^{J K}$ is the mean of $\hat{Y}_{(k)}^{J K}$.

\section{A simulation study}

In this section, the composite estimator which is the linear combination of the two stage cluster and the ratio estimators is compared with the other two estimators to improve the estimate accuracy for the stratified two stage cluster simple random sampling with different cluster sizes. For simplicity, we assume the number of strata is $L=1$.

For the simulation study, the number of clusters in population is $N=N_{1}=500$, the number of sample clusters is $n=n_{1}=20,40$ and the number of sample elements in cluster is $m_{h i}=m_{1 i}=5,10$. First the normal data are generated with difference size of cluster unit, $M_{h i}=M_{1 i}$. Also for existing variation of means between clusters, we consider different mean $m_{Y_{i}}$ in normal distribution. Lastly, for considering changes of population size $M_{1}$ at the time of survey period, we generate values of $M_{1}$ from normal distribution with mean $M_{\text {true }}=\sum_{i=1}^{N} M_{1 i}$ and variance $\sigma_{M_{1}}^{2}$. Following are the simulation set up and the values used for simulation.

(1) $M_{1 i} \stackrel{i i d}{\sim} N\left(\bar{M}_{1}, \sigma_{\bar{M}_{1}}^{2}\right), \quad\left(\bar{M}_{1}, \sigma_{\bar{M}_{1}}\right)=(30,4),(60,4),(60,8)$.

(2) $M_{1} \stackrel{\text { iid }}{\sim} N\left(M_{\text {true }}, \sigma_{M_{1}}^{2}\right), \quad \mathrm{CV}_{1}=\sigma_{M_{1}} / M_{\text {true }}=0,0.01,0.02,0.03$.

(3) $m_{Y_{i}} \stackrel{i i d}{\sim} N\left(\mu_{Y}, \sigma_{m Y}^{2}\right), \quad\left(\mu_{Y}, \sigma_{m Y}\right)=(200,24)$.

(4) $y_{1 i j} \stackrel{i i d}{\sim} N\left(m_{Y_{i}}, \sigma_{Y}^{2}\right), \quad \sigma_{Y}=10$.

We also use root mean squared error (RMSE), Bias, absolute bias (ABias) for the comparison statistics defined by

$$
\operatorname{RMSE}=\sqrt{\frac{1}{R} \sum_{r=1}^{R}\left(\hat{Y}^{(r)}-Y\right)^{2}}
$$


Table 1: Comparison results with $\left(\bar{M}_{1}, \sigma_{\bar{M}_{1}}\right)=(30,4)$

\begin{tabular}{|c|c|c|c|c|c|c|c|c|c|c|c|}
\hline \multirow{2}{*}{$n$} & \multirow{2}{*}{$m_{h_{i}}$} & \multirow{2}{*}{$\begin{array}{l}\mathrm{CV}_{1} \\
(\%)\end{array}$} & \multicolumn{3}{|c|}{ Bias } & \multicolumn{3}{|c|}{ Abias } & \multicolumn{3}{|c|}{ RMSE } \\
\hline & & & ratio & cluster & com & ratio & cluster & com & ratio & cluster & com \\
\hline \multirow{8}{*}{20} & \multirow{4}{*}{5} & 0 & -5653 & -7574 & -6897 & 61365 & 101170 & 76930 & 77050 & 125924 & 96350 \\
\hline & & 1 & -312 & -6488 & -2963 & 67699 & 102045 & 79506 & 86071 & 127995 & 99599 \\
\hline & & 2 & 2441 & 1268 & 1653 & 77656 & 91127 & 77482 & 98211 & 112349 & 96215 \\
\hline & & 3 & -5318 & -305 & -1894 & 95540 & 84619 & 78215 & 120844 & 106776 & 98430 \\
\hline & \multirow{4}{*}{10} & 0 & 435 & 3219 & 1734 & 63175 & 86358 & 71569 & 79513 & 109888 & 91192 \\
\hline & & 1 & -4181 & -484 & -2323 & 68463 & 89846 & 74357 & 85076 & 111740 & 93145 \\
\hline & & 2 & 3943 & 1785 & 2909 & 76286 & 86361 & 74568 & 95774 & 110127 & 93214 \\
\hline & & 3 & 3917 & 2067 & 3918 & 90996 & 87383 & 76466 & 114142 & 108929 & 96018 \\
\hline \multirow{8}{*}{40} & \multirow{4}{*}{5} & 0 & -3387 & -2708 & -3163 & 42639 & 55372 & 46015 & 53180 & 69039 & 56768 \\
\hline & & 1 & -1303 & -1938 & -1692 & 50192 & 58119 & 49758 & 63363 & 73246 & 62801 \\
\hline & & 2 & -1873 & -2795 & -1878 & 63968 & 59279 & 54812 & 80239 & 74044 & 69342 \\
\hline & & 3 & 1307 & 3439 & 3257 & 84915 & 63190 & 64886 & 106087 & 78810 & 81292 \\
\hline & \multirow{4}{*}{10} & 0 & 136 & 3384 & 1507 & 43763 & 59919 & 47779 & 55228 & 74335 & 59948 \\
\hline & & 1 & 1983 & 261 & 1074 & 50405 & 56557 & 48926 & 63690 & 71156 & 61293 \\
\hline & & 2 & -32 & 3881 & 2199 & 64503 & 58688 & 54718 & 80708 & 73364 & 68497 \\
\hline & & 3 & 578 & 504 & 1549 & 80686 & 60774 & 62346 & 101530 & 76981 & 78455 \\
\hline
\end{tabular}

Abias = absolute bias, RMSE = root mean squared error.

Table 2: Comparison results with $\left(\bar{M}_{1}, \sigma_{\bar{M}_{1}}\right)=(60,4)$

\begin{tabular}{|c|c|c|c|c|c|c|c|c|c|c|c|}
\hline \multirow{2}{*}{$n$} & \multirow{2}{*}{$m_{h_{i}}$} & \multirow{2}{*}{$\begin{array}{l}\mathrm{CV}_{1} \\
(\%)\end{array}$} & \multicolumn{3}{|c|}{ Bias } & \multicolumn{3}{|c|}{ Abias } & \multicolumn{3}{|c|}{ RMSE } \\
\hline & & & ratio & cluster & com & ratio & cluster & com & ratio & cluster & com \\
\hline \multirow{8}{*}{20} & \multirow{4}{*}{5} & 0 & 2738 & -719 & 544 & 129244 & 149364 & 139756 & 162020 & 187351 & 175130 \\
\hline & & 1 & -1885 & -5480 & -3629 & 138010 & 164342 & 147252 & 170317 & 205318 & 183362 \\
\hline & & 2 & 4821 & -74 & 2827 & 161636 & 168591 & 152781 & 202792 & 209229 & 190531 \\
\hline & & 3 & 8014 & 552 & 5989 & 188795 & 146808 & 143700 & 238981 & 183651 & 180516 \\
\hline & \multirow{4}{*}{10} & 0 & -5629 & -4427 & -5035 & 125727 & 139736 & 133095 & 156323 & 173100 & 164868 \\
\hline & & 1 & -12732 & -12519 & -12479 & 134527 & 138234 & 133149 & 166828 & 172477 & 165391 \\
\hline & & 2 & 3199 & 447 & 2194 & 152801 & 136211 & 133179 & 192956 & 170680 & 167668 \\
\hline & & 3 & -1471 & 2703 & 3639 & 188964 & 138715 & 137314 & 234252 & 171749 & 171321 \\
\hline \multirow{8}{*}{40} & \multirow{4}{*}{5} & 0 & 2473 & 3068 & 2624 & 91554 & 95604 & 91590 & 114135 & 121122 & 115838 \\
\hline & & 1 & -1467 & 4726 & 2419 & 94104 & 98611 & 90347 & 119672 & 124781 & 114614 \\
\hline & & 2 & -5675 & -763 & -1864 & 135500 & 100956 & 104694 & 168242 & 124306 & 129777 \\
\hline & & 3 & -4411 & -176 & 1172 & 167519 & 95438 & 109145 & 208048 & 120531 & 135661 \\
\hline & \multirow{4}{*}{10} & 0 & -473 & 779 & -74 & 89211 & 98252 & 92475 & 111957 & 122360 & 115568 \\
\hline & & 1 & -769 & -884 & -913 & 96250 & 95815 & 91510 & 119786 & 119180 & 113429 \\
\hline & & 2 & 2785 & 2044 & 3635 & 132502 & 98426 & 101113 & 164583 & 122046 & 126581 \\
\hline & & 3 & 4458 & -264 & 4092 & 163370 & 92351 & 105927 & 204709 & 117070 & 133566 \\
\hline
\end{tabular}

Abias = absolute bias, RMSE $=$ root mean squared error.

$$
\begin{aligned}
\text { Bias } & =\frac{1}{R} \sum_{r=1}^{R}\left(\hat{Y}^{(r)}-Y\right), \\
\text { ABias } & =\frac{1}{R} \sum_{r=1}^{R}\left|\hat{Y}^{(r)}-Y\right| .
\end{aligned}
$$

Here we use replication number, $R=1,000$ and the results are tabulated in Table 1 to Table 3 . From Table 1 to Table 3, we can see the similar trend of results. First of all, based on Bias, we cannot see any pattern by changes in the numbers of sample clusters or elements of each cluster. The three estimators are all unbiased. Now when we change the number of sample clusters, 20 to 40 which means, the number of sample clusters is increasing, the results of Abias and RMSE show that all 
Table 3: Comparison results with $\left(\bar{M}_{1}, \sigma_{\bar{M}_{1}}\right)=(60,8)$

\begin{tabular}{|c|c|c|c|c|c|c|c|c|c|c|c|}
\hline \multirow{2}{*}{$n$} & \multirow{2}{*}{$m_{h_{i}}$} & \multirow{2}{*}{$\begin{array}{l}\mathrm{CV}_{1} \\
(\%)\end{array}$} & \multicolumn{3}{|c|}{ Bias } & \multicolumn{3}{|c|}{ Abias } & \multicolumn{3}{|c|}{ RMSE } \\
\hline & & & ratio & cluster & com & ratio & cluster & com & ratio & cluster & com \\
\hline \multirow{8}{*}{20} & \multirow{4}{*}{5} & 0 & 2091 & -224 & 454 & 126682 & 173960 & 145137 & 160600 & 223836 & 186664 \\
\hline & & 1 & -18738 & -13050 & -15411 & 137025 & 194506 & 157803 & 169711 & 239948 & 194287 \\
\hline & & 2 & -2874 & -4180 & -3381 & 157295 & 174411 & 150204 & 200333 & 221292 & 191979 \\
\hline & & 3 & 16182 & 6295 & 11413 & 194807 & 169451 & 159063 & 243860 & 212264 & 198785 \\
\hline & \multirow{4}{*}{10} & 0 & -5714 & -4375 & -6311 & 123797 & 163102 & 139152 & 155073 & 206022 & 174892 \\
\hline & & 1 & 347 & 1286 & 859 & 134821 & 172857 & 147388 & 170415 & 215237 & 182762 \\
\hline & & 2 & 972 & -501 & 1154 & 164665 & 173700 & 154188 & 205641 & 215881 & 192029 \\
\hline & & 3 & -8868 & -10257 & -8500 & 191113 & 175508 & 161688 & 240872 & 220967 & 201210 \\
\hline \multirow{8}{*}{40} & \multirow{4}{*}{5} & 0 & 3127 & -493 & 1293 & 89605 & 121985 & 97760 & 110815 & 151083 & 120078 \\
\hline & & 1 & -1255 & 5618 & 1833 & 98782 & 121432 & 98211 & 123481 & 152000 & 123374 \\
\hline & & 2 & -1760 & -12 & 12 & 126862 & 126322 & 109212 & 160940 & 157566 & 139188 \\
\hline & & 3 & -2221 & -575 & 1250 & 165024 & 127531 & 124456 & 206835 & 158103 & 157917 \\
\hline & \multirow{4}{*}{10} & 0 & 2234 & 1055 & 1960 & 91919 & 130454 & 100770 & 115739 & 163531 & 127420 \\
\hline & & 1 & 6628 & 2888 & 5196 & 103656 & 125007 & 103859 & 129933 & 156408 & 130869 \\
\hline & & 2 & -3762 & -9226 & -4681 & 131463 & 121479 & 112264 & 164179 & 152589 & 139289 \\
\hline & & 3 & 550 & 1255 & 2797 & 177316 & 122268 & 130812 & 216391 & 153856 & 159909 \\
\hline
\end{tabular}

Abias = absolute bias, RMSE = root mean squared error.

estimators improve the accuracy of estimates. However, obviously increasing $m_{h_{i}}, 5$ to 10 , does shows little improvement.

It is clear that if $\mathrm{CV}_{1}$ gets larger, then the ratio estimator rapidly deteriorates. Obviously the results of the two stage cluster estimator do not change because of not using the information of the size of population, $M_{h}=M_{1}$. Now for the case of $\mathrm{CV}_{1}=0$, with known and accurate population information, the ratio estimator is the best. However, if $\mathrm{CV}_{1}$ is greater than 0.01 then the two stage cluster estimator is better than the ratio estimator.

However, the suggested composite estimator shows stability on every case. Especially, the suggested composite estimator has relative merit for the case of $\mathrm{CV}_{1}=0.01$. That is, the suggested estimator is at least the same as the better one when based on Abias and RMSE statistics. Furthermore, when $\mathrm{CV}_{1}$ gets large, the ratio estimator rapidly deteriorates. However, the suggested estimator still gives stable results. Also, the two stage cluster estimator has a large bias compared to the suggested estimator (relatively).

Consequently, the ratio estimator is the best if we do have known and accurate information on population. However, knowing the accurate information about the population at the survey period is not quite possible and difficult. Therefore the suggested composite estimator will be best when the population is changed but not knowing the exact information.

\section{A real data analysis}

In this section, we use Taxi Company data from 170 taxi companies in Korea. The data includes the variables: region, company name, travel distance/transfer distance and we are interested in the travel distance variable. For this real data analysis, we adopt the Salvati et al. (2010) method for generating pseudo population. With 170 surveyed taxi company data, the pseudo population is generated by re-samples of clusters, with a replacement 30 times. That means we have 5,100 clusters in pseudo population. The administrative district are made of 2 strata. Then the number of clusters in each stratum, $N_{j}$ and the number of elements in each cluster, $M_{h i}$ are obtained. Among these, we deleted $M_{h i}$ which is greater than 150 or less than 7 because in practice the differences between $M_{h i}$ are hardly large. 
Table 4: Comparison results for real data analysis (Stratum 1)

\begin{tabular}{|c|c|c|c|c|c|c|c|c|c|c|c|}
\hline \multirow{2}{*}{$n$} & \multirow{2}{*}{$m_{h_{i}}$} & \multirow{2}{*}{$\begin{array}{l}\mathrm{CV}_{1} \\
(\%)\end{array}$} & \multicolumn{3}{|c|}{ Bias } & \multicolumn{3}{|c|}{ Abias } & \multicolumn{3}{|c|}{ RMSE } \\
\hline & & & ratio & cluster & com & ratio & cluster & com & ratio & cluster & com \\
\hline \multirow{8}{*}{15} & \multirow{4}{*}{3} & 0 & -250 & -3390 & -1671 & 7130 & 9513 & 8234 & 8877 & 11889 & 10336 \\
\hline & & 3 & 238 & -2794 & -1334 & 7595 & 8722 & 7601 & 9642 & 11019 & 9624 \\
\hline & & 5 & 271 & -2312 & -682 & 9024 & 9172 & 8232 & 11335 & 11510 & 10405 \\
\hline & & 10 & 806 & -2624 & -443 & 12863 & 8952 & 8989 & 16165 & 11276 & 11414 \\
\hline & \multirow{4}{*}{5} & 0 & -266 & -3037 & -1573 & 5745 & 8273 & 6751 & 7255 & 10456 & 8492 \\
\hline & & 3 & 136 & -2224 & -992 & 6583 & 7905 & 6816 & 8234 & 9946 & 8539 \\
\hline & & 5 & 79 & -2960 & -854 & 12692 & 7870 & 8247 & 15828 & 9954 & 10481 \\
\hline & & 10 & -297 & -2666 & -1110 & 7943 & 8017 & 6947 & 9916 & 10085 & 8752 \\
\hline \multirow{8}{*}{30} & \multirow{4}{*}{3} & 0 & 32 & -1619 & -1050 & 4404 & 4985 & 4665 & 5495 & 6242 & 5818 \\
\hline & & 3 & 517 & -1404 & -745 & 5380 & 4716 & 4608 & 6860 & 5997 & 5851 \\
\hline & & 5 & 325 & -1644 & -865 & 7054 & 4871 & 4953 & 8924 & 6245 & 6346 \\
\hline & & 10 & -816 & -1805 & -1174 & 11905 & 4931 & 6024 & 14941 & 6262 & 7986 \\
\hline & \multirow{4}{*}{5} & 0 & 56 & -1635 & -1149 & 3265 & 3775 & 3487 & 4094 & 4754 & 4375 \\
\hline & & 3 & -37 & -1499 & -1044 & 4569 & 3764 & 3672 & 5775 & 4752 & 4565 \\
\hline & & 5 & 326 & -1743 & -997 & 6441 & 3925 & 3959 & 8168 & 4920 & 5017 \\
\hline & & 10 & 605 & -1584 & -505 & 11675 & 3744 & 4843 & 14606 & 4800 & 6511 \\
\hline
\end{tabular}

Abias = absolute bias, RMSE = root mean squared error.

Table 5: Comparison results for real data analysis (Stratum 2)

\begin{tabular}{|c|c|c|c|c|c|c|c|c|c|c|c|}
\hline \multirow{2}{*}{$n$} & \multirow{2}{*}{$m_{h_{i}}$} & \multirow{2}{*}{$\begin{array}{l}\mathrm{CV}_{1} \\
(\%)\end{array}$} & \multicolumn{3}{|c|}{ Bias } & \multicolumn{3}{|c|}{ Abias } & \multicolumn{3}{|c|}{ RMSE } \\
\hline & & & ratio & cluster & com & ratio & cluster & com & ratio & cluster & com \\
\hline \multirow{8}{*}{15} & \multirow{4}{*}{3} & 0 & -1662 & 1635 & -317 & 7353 & 8819 & 7906 & 9429 & 11240 & 10049 \\
\hline & & 3 & -1015 & 2166 & 689 & 7822 & 8884 & 8155 & 9949 & 11047 & 10204 \\
\hline & & 5 & -1258 & 1843 & 384 & 9360 & 9317 & 8432 & 11844 & 11535 & 10660 \\
\hline & & 10 & -325 & 1849 & 406 & 12348 & 8360 & 8816 & 15759 & 10581 & 11607 \\
\hline & \multirow{4}{*}{5} & 0 & -1364 & 1951 & 221 & 6574 & 8249 & 7017 & 8410 & 10378 & 8851 \\
\hline & & 3 & -1060 & 2218 & 64 & 7377 & 8359 & 7121 & 9339 & 10448 & 9090 \\
\hline & & 5 & -935 & 2549 & 842 & 8126 & 8499 & 7444 & 10469 & 10615 & 9383 \\
\hline & & 10 & -975 & 2051 & 1142 & 12961 & 8321 & 9004 & 16500 & 10323 & 11344 \\
\hline \multirow{8}{*}{30} & \multirow{4}{*}{3} & 0 & -909 & -387 & -559 & 4058 & 4293 & 4238 & 5174 & 5410 & 5371 \\
\hline & & 3 & -732 & -551 & -587 & 5090 & 4326 & 4366 & 6403 & 5372 & 5504 \\
\hline & & 5 & -530 & -628 & -384 & 6612 & 4436 & 4777 & 8374 & 5592 & 6118 \\
\hline & & 10 & -1898 & -963 & -852 & 11508 & 4236 & 5549 & 14338 & 5367 & 7552 \\
\hline & \multirow{4}{*}{5} & 0 & -1278 & -954 & -1056 & 3304 & 3566 & 3449 & 4192 & 4452 & 4327 \\
\hline & & 3 & -997 & -678 & -730 & 4498 & 3527 & 3561 & 5723 & 4501 & 4575 \\
\hline & & 5 & -776 & -646 & -629 & 6350 & 3640 & 3971 & 8039 & 4621 & 5173 \\
\hline & & 10 & -1161 & -718 & -464 & 11630 & 3586 & 5098 & 14489 & 4640 & 7158 \\
\hline
\end{tabular}

Abias = absolute bias, RMSE = root mean squared error.

From each stratum, sample clusters with $n_{h}=15,30$ are selected and from each cluster, samples with $m_{h i}=3,5$ are selected. Three estimators stated in Section 3 are calculated and the results are compared based on the comparison statistics in Section 4. We use CV $=0,0.03,0.05$ and 0.1 to consider the changes on population. The number of replications is 1,000 and the results are in Table 4 and Table 5.

Both Tables 4 and 5 show similar results. First, based on Bias, we cannot see any trend or pattern because three estimators are all unbiased. However, based on Abias and RMSE, the results vary depending on the value of CV. Especially from Table 4 in case of $n=15$, the results of the two stage cluster and the ratio estimates are switched with respect to RMSE in between CV $=0.05$ and $C V=0.1$. However, the results of the case of $n=30$, are switched between $C V=0.03$ and $\mathrm{CV}=0.05$. The suggested composite estimator also shows stable and relatively good results. Table 5 
shows similar results to Table 4 .

\section{Summary and conclusion}

When the total number of population elements is unknown, the two stage cluster estimator should be used to estimate the total. However, the ratio estimator is recommended if the total number of population elements is known and accurate. The ratio estimator or the two stage cluster estimator can be used when the total number of population elements is known but not accurate. At this point, the choice of estimators is depending on the accuracy about information of population and on the number of cluster elements. However, those terms cannot be assured in general. Therefore, we suggest a composite estimator which is a linear combination of the two stage cluster and the ratio estimators.

There exists a time difference between sampling frame and survey period. Also, cluster sampling designs are usually used to reduce the survey costs. Therefore, the number of population elements is not known in most cases and the sizes of each cluster are not the same. For that case, the suggested composite estimator will give more stable and better estimates compared with two other estimators under those conditions.

\section{Acknowledgement}

This study was supported by Hankuk University of Foreign Studies research fund (2015).

\section{References}

Cochran WG (1977). Sampling Technique, John Wiley and Sons, New York.

Hwang HJ and Shin KI (2013). An improved composite estimator for cut-off sampling, Communications for Statistical Applications and Method, 20, 367-376.

Lee SE, Jin Y, and Shin KI (2015). A Note on complex two-phase sampling with different sampling units of each phase, Communications for Statistical Applications and Method, 22, 435-443.

Quenouille LLE MH (1949). The joint distribution of serial correlation coefficients, The Annals of Mathematical Statistics, 20, 561-571.

Rao JNK (2003). Small Area Estimation, Wiley-Interscience.

Salvati N, Chandra H, Ranalli MG, and Chambers R (2010). Small area estimation using a nonparametric model-based direct estimator, Computational Statistics and Data Analysis, 54, 2159 2171.

Tukey JW (1958). Bias and confidence in not quite large samples, Annals of Mathematical Statistics, 29, 614 .

Wolter KM (1985). Introduction to Variance Estimation, Springer, New York. 